\title{
Case Report: Simultaneous Injury of Mid-shaft Clavicle Fracture and Type IV Acromioclavicular Joint Dislocation
}

\author{
Amir Sobhani Eraghi' ${ }^{1}$ (D), Mehdi Moghtadaei ${ }^{1}$ (D) Iman Azizpour $^{1}$ (D), Mikaiel Hajializade ${ }^{1^{*}}$ (D)
}

1. Department of Orthopedic Surgery, Rasoul-e Akram Hospital, Iran University of Medical Sciences, Tehran, Iran.

Citation Sobhani Eraghi A, Moghtadaei M, Azizpour I, Hajializade M. Simultaneous Injury of Mid-shaft Clavicle Fracture and Type IV Acromioclavicular Joint Dislocation. Journal of Research in Orthopedic Science. 2020; 7(1):35-40. http://dx.doi. org/10.32598/JROSJ.7.1.35

: http://dx.doi.org/10.32598/JROSJ.7.1.35

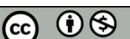

Article info:

Received: 13 May 2019

Revised: 25 Jun 2019

Accepted: 25 Nov 2019

Available Online: 01 Feb 2020

Keywords:

Mid-shaft

clavicle fracture,

Acromioclavicular joint

dislocation, Internal

fixation, EndoButton,

Simultaneous

\begin{abstract}
A B S T RA C T
Background: Combined injuries of Mid-shaft clavicle fracture and acromioclavicular (AC) joint dislocation are rare, and only a few cases have been reported. Several treatment options including surgical, conservative and hybrid approach have been described. Yet, there is no consensus regarding the optimal management approach for this injury.

Objectives: Here we reported a case of Mid-shaft clavicle fracture with associated type IV AC joint dislocation in a 29-year-old male following a cycling accident.

Methods: Both parts of the injury were fixed surgically. Meanwhile, the patient did not follow the postoperative protocol and started the heavy sports activities one month after the surgery.

Results: The patient showed up 6weeks after the surgery with slightly uncorrected AC joint. However, he was satisfied with the results and accordingly no intervention was done for the correction of the $\mathrm{AC}$ joint.

Conclusion: Our case reveals the importance of adherence to the postoperative protocol in this combined and challenging injury and we recommend surgical fixation for such injury.
\end{abstract}

\section{Introduction}

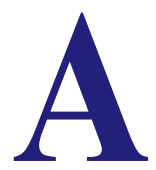

Clavicle fracture is one of the most common skeletal fractures accounting for $2 \%-5 \%$ of all adult fractures [1]. An incidence rate of 29-64 cases per 100000 has been reported for the clavicle fracture [2]. Sports injuries in high-demanding individuals are responsible for almost half of the clavicle fractures [3]. Although fracture might occur in any part of the clavi- cle, the mid-shaft is the most frequent site (nearly $80 \%$ of fractures) as there are no strong ligaments and muscle coverage in this area, and the curved bone is weak as well [4]. Optimal treatment of clavicle fracture remains controversial, and it depends on the type and displacement of fracture [5].

Acromioclavicular (AC) joint dislocation is also one of the most common injuries of the shoulder and accounts for nearly $9 \%$ of all shoulder girdle injuries [6]. Simi-

* Corresponding Author:

Mikael Hajializade, MD.

Address: Department of Orthopedic Surgery, Rasoul-e Akram Hospital, Iran University of Medical Sciences, Tehran, Iran.

Phone: +98 (21) 66515001

E-mail: michaelalizadeh@gmail.com 
lar to the clavicle fracture, AC joint dislocation mostly occurs following sports activities with an incidence rate of 1.8 cases per 10000 people-years [7]. Although surgical treatment is mostly indicated for injuries of grades IV or higher, their nonsurgical treatment outcomes are comparable to the surgical procedure, according to some studies [8].

Dislocation of the AC joint in combination with the fracture of the distal end of the clavicle is also frequent. However, the dislocation of the $\mathrm{AC}$ joint combined with the fracture of the middle third of the clavicle is rare, and only a few reports are available in this regard. Thus, the diagnosis and treatment of these cases could be challenging $[9,10]$.

Here we report a case of AC joint type IV dislocation combined with the fracture of the middle third of the clavicle in a 29-year-old male following a cycling accident that was successfully treated with surgery.

\section{Case Presentation}

A 29-year-old male with a left shoulder injury following a cycling accident was referred to the emergency department of our center. On clinical examination, ecchymosis and swelling were seen in the affected area. Besides, a marked tenderness was noticed at the mid-clavicle. The neurovascular status of the shoulder was normal. Yet, he was unable to move his shoulder actively.
Radiographic evaluation of the involved shoulder revealed a displaced fracture in the middle third of the clavicle. Type IV dislocation of the AC joint was detected in CT scan (Figure 1), and an elective surgical treatment was planned for the patient one week later. Briefly, after general anesthesia and placing the patient in a beach-chair position, a longitudinal incision was made extending laterally over the $\mathrm{AC}$ joint to expose the clavicle. After the reduction of clavicle fracture, the first fixation of the clavicle was performed with an anatomical plate in the superior surface of the clavicle. The AC joint was also reduced and fixed with the EndoButton technique from the hole of anatomical plate to the coracoid, and finally, deltotrapezial fascia was reconstructed.

After the operation, a shoulder sling was applied for 4 weeks. The patient was instructed for a gentle range of motion up to the shoulder level. Moreover, the patient was ordered not to perform heavy sports activities for 3 months. Clinical and radiological follow-up was planned at weeks 2 and 4, and months 3, 6, and 12. However, the patient did not attend the follow-up sessions regularly and missed the first two follow-up sessions. The patient showed up 6 weeks after the surgery for the first time with a slight loss of reduction of $\mathrm{AC}$ joint compared with what achieved immediately after the operation (Figure 2).

At the history-taking process, he admitted starting heavy sports activities (bodybuilding) 4 weeks after the operation, thinking that it would help his muscle to re-

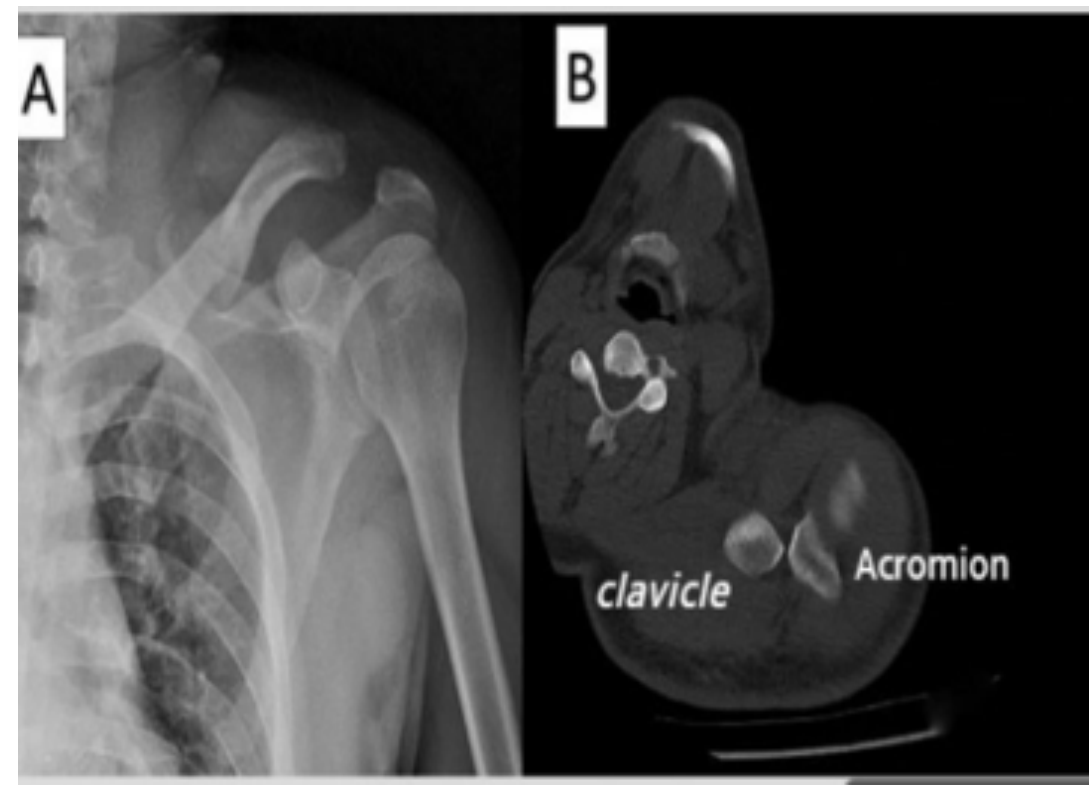

Orthopedic Science

Figure 1. Clavicle Fracture and Type IV A-c Joint Dislocation

A. Preoperative anteroposterior radiograph of the shoulder showing the fracture of middle third of the clavicle and type IV dislocation of acromioclavicular joint; B. Preoperative axial CT scan of patient showing type IV dislocation 


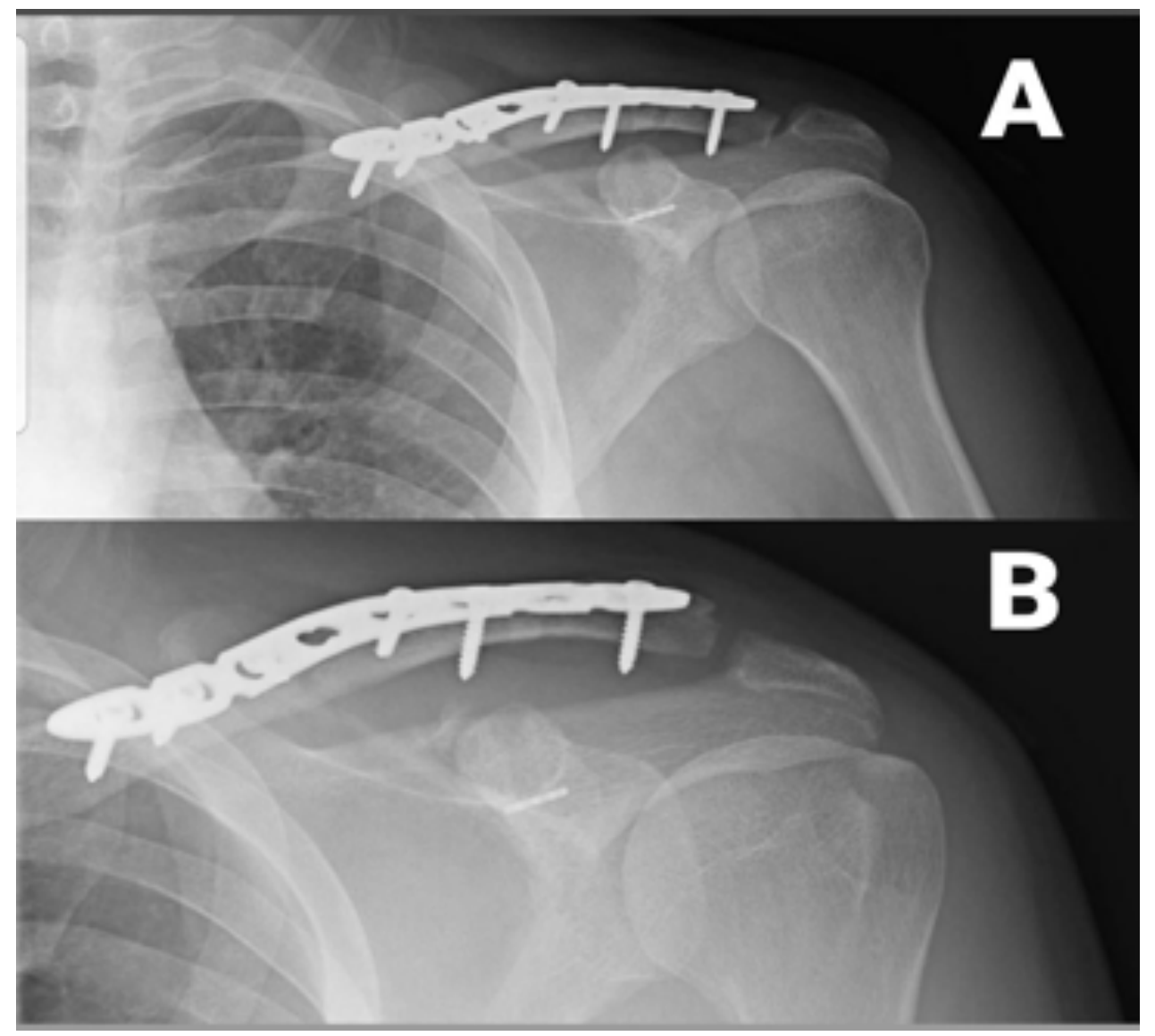

Orthopedic Science

Figure 2. Fixation of Clavicle Fracture and A-c Joint Dislocation

A. Anteroposterior radiograph of the shoulder after surgery showing the fixation of the fracture with an anatomical plaque and fixation of dislocation with EndoButton; B. three months after the operation showing a slightly undercorrected acromioclavicular joint caused by early heavy sports activity

gain power and accelerate his recovery, although we had ordered him not to do that. Twelve weeks after the surgery, his shoulder range of motion was normal, and the patient was satisfied with the results. Thus, no intervention was done for the correction of the uncorrected AC joint. The patient was followed up for one year, and at the end of the year, he had the same radiographic and clinical findings as week 12.

\section{Discussion}

Combined injuries involving an ipsilateral fracture of the clavicle mid-shaft and AC joint dislocation is rare. There is no consensus over the best management of these injuries. Thus, a variety of treatment approaches have been used, including nonsurgical, surgical, and hybrid management of the two distinct parts of this injury. Meanwhile, the most appropriate approach depends on the level of AC joint instability [11], and the surgical fixation is recommended for dislocation type IV or higher.
In this report, we presented a case of combined ipsilateral fracture of the middle-third of clavicle and AC joint dislocation that occurred following a cycling accident. The patient underwent surgical treatment, and his displaced clavicle fracture and $\mathrm{AC}$ joint dislocation were both reduced and fixed, and no instability was detected postoperatively. These results reveal that open reduction and internal fixation are a reliable treatment of the combined displaced fracture of the mid-shaft clavicle and AC joint dislocation.

Woolf et al. reported a case of mid-shaft clavicle fracture with type IV AC joint dislocation. The injury occurred during throwing out from a high-speed motor vehicle collision. Internal fixation of each injury was selected as the preferred treatment approach, and the patient had a successful result at the final evaluation [11]

Tidwell et al. reported a case of AC dislocation with a displaced fracture of the middle third of the clavicle in 
a 19-year-old male while driving an all-terrain vehicle. The injury was managed with open reduction and internal fixation of the fracture, open reduction of the AC joint dislocation and coracoclavicular screw fixation. The patient returned to normal daily activities and was satisfied with the result [12].

Wurtz et al. also reported this combined injury in 4 other cases. The injury occurred following horse-back falling in two cases, cycling accident in one case, and motor-vehicle-accident in the other one. Three out of four cases were diagnosed with type IV dislocation and underwent surgical fixation. This approach led to the successful and asymptomatic range of motion. The other patient was diagnosed with type II dislocation and was treated nonoperatively and ended up with a painless outcome and full range of motion [13].

Although surgical treatment is recommended for this combined injury, especially for dislocation types of IV or higher, nonsurgical management has also been reported, even in the presence of type IV dislocation. John and Simonian reported a case of combined mid-clavicle fracture and type IV dislocation in a hockey player. Both injuries were treated nonoperatively with good results. The patient returned to his full range of activity 14 weeks after injury [14]. Although this study revealed an acceptable outcome following the nonoperative management of mid-clavicle fracture with type IV dislocation, the unfavorable outcome has also been reported even at the presence of lower types of AC joint dislocation. Heinz and Misamore reported a case of a cyclist who sustained the combined injury of mid-clavicle fracture and AC dislocation (type III) during a race.

The injury was managed conservatively with a clavicle brace for 5 weeks. However, follow-up radiographs of the shoulder revealed a wide AC separation along with the superior displacement of the clavicle [10]. Hybrid management (surgical fixation of only one part of the injury) has also been reported. Lancourt described the case of a patient with a displaced mid-shaft clavicle fracture and type V AC dislocation. The AC dislocation was fixed surgically. Meanwhile, the clavicle fracture was managed nonoperatively.

After 3 years of follow-up, good radiographic and clinical outcomes were obtained [15]. Therefore, there is no clear-cut answer for the optimal management of mid-shaft clavicle fracture and a type IV AC dislocation. While all types of management (conservative, surgical, hybrid) could result in an acceptable outcome, surgical fixation of both parts of the injury seems to be the most popular approach in dislocations type IV or more, and it seems that the patient returns to regular activity and work is faster in surgery than conservative treatment.

\section{Conclusion}

Whatever type of treatment is selected, it should be noted that adherence to the postoperative protocol is necessary for a successful functional outcome. In our case, the patient did not observe the postoperative protocol and started heavy sports activities (bodybuilding) 4 weeks after the surgery. The patient also did not attend the follow-up sessions regularly and showed up with a slight loss of reduction of AC joint, 6 weeks after the surgery. Although the patient was satisfied with the results, this observation highlights the importance of regular radiographic and clinical follow-ups, as well as adherence to the postoperative protocol.

\section{Ethical Considerations}

\section{Compliance with ethical guidelines}

All ethical principles were considered in this article. The patient was informed that his data and pictures would be submitted for publi cation and gave his consent.

\section{Funding}

This research did not receive any specific grant from funding agencies in the public, commercial, or not-forprofit sectors

\section{Authors' contributions}

Conceptualization, supervision: Amir Sobhani Eraghi, Mehdi Moghtadaei; surgeon: Amir Sobhani Eraghi; Visualization, assisted surgeon: Iman Azizpour, Mikaiel Hajializade; Writing - original draft, data curation, methodology: Mikaie Hajializade.

\section{Conflict of interest}

The authors declared no conflict of interest.

\section{References}

[1] Postacchini F, Gumina S, De Santis P, Albo F. Epidemiology of clavicle fractures. J Shoulder Elbow Surg. 2002; 11(5):452-6. [DOI:10.1067/mse.2002.126613] [PMID] 
[2] Robinson CM. Fractures of the clavicle in the adult: Epidemiology and classification. J Bone Joint Surg Br. 1998; 80(3):476-84. [DOI:10.1302/0301-
[D 620X.80B3.0800476] [PMID]

[3] Toogood P, Horst P, Samagh S, Feeley BT. Clavicle fractures: a review of the literature and update on treatment. Phys Sportsmed. 2011; 39(3):142-50. [DOI:10.3810/ psm.2011.09.1930] [PMID]

[4] Rowe CR. An atlas of anatomy and treatment of midclavicular fractures. Clin Orthop Relat Res. 1968; 58:29-42. [DOI:10.1097/00003086-196805000-00006] [PMID]

[5] Kihlström C, Möller M, Lönn K, Wolf O. Clavicle fractures: Epidemiology, classification and treatment of 2 422 fractures in the Swedish fracture register; an observational study. BMC Musculoskelet Disord. 2017; 18(1):82. [DOI:10.1186/s12891-017-1444-1] [PMID] [PMCID]

[6] Mazzocca AD, Arciero RA, Bicos J. Evaluation and treatment of acromioclavicular joint injuries. Am J Sports Med. 2007; 35(2):316-29. [DOI:10.1177/0363546506298022] [PMID]

[7] Chillemi C, Franceschini V, Dei Giudici L, Alibardi A, Salate Santone F, Ramos Alday LJ, et al. Epidemiology of isolated acromioclavicular joint dislocation. Emerg Med Int. 2013; 2013:171609. [DOI:10.1155/2013/171609] [PMID] [PMCID]

[8] Boffano M, Mortera S, Wafa H, Piana R. The surgical treatment of acromioclavicular joint injuries. EFORT Open Rev. 2017; 2(10):432-7. [DOI:10.1302/2058-5241.2.160085] [PMID] [PMCID]

[9] Mohammed KD, Stachiw D, Malone AA. Type IV acromioclavicular joint dislocation associated with a mid-shaft clavicle malcunion. Int J Shoulder Surg. 2016; 10(1):37-40. [DOI:10.4103/0973-6042.174518] [PMID] [PMCID]

[10] Heinz WM, Misamore GW. Mid-shaft fracture of the clavicle with grade III acromioclavicular separation. J Shoulder Elbow Surg. 1995; 4(2):141-2. [DOI:10.1016/S10582746(05)80069-2] [PMID]

[11] Woolf SK, Valentine BJ, Barfield WR, Hartsock LA Middle-third clavicle fracture with associated type IV acromioclavicular separation: Case report and literature review. J Surg Orthop Adv. 2013; 22(2):183-6. [DOI:10.3113/ JSOA.2013.0183] [PMID]

[12] Tidwell JE, Kennedy PM, McDonough EB. Concurrent treatment of a middle-third clavicle fracture and type IV acromioclavicular dislocation. Am J Orthop (Belle Mead NJ). 2014; 43(11):E275-8. [PMID]

[13] Wurtz LD, Lyons FA, Rockwood CA Jr. Fracture of the middle third of the clavicle and dislocation of the acromioclavicular joint. A report of four cases. JBJS. 1992; 74(1):133-7. [DOI:10.2106/00004623-199274010-00018]

[14] Juhn MS, Simonian PT. Type VI acromioclavicular separation with middle-third clavicle fracture in an ice hockey player. Clin J Sport Med. 2002; 12(5):315-7. [DOI:10.1097/00042752-200209000-00011] [PMID]

[15] Lancourt JE. Acromioclavicular dislocation with adjacent clavicular fracture in a horseback rider. A case report. Am
J Sports Med. 1990; 18(3):321-2. [DOI:10.1177/0363546590 01800317] [PMID] 
This Page Intentionally Left Blank 